\title{
As-Cast Structure and Metallurgical Inheritance of High Nitrogen Austenitic Stainless Steel
}

\author{
Alexander A. Kazakov ${ }^{1}$, Eduard Kolpishon ${ }^{2}$, Aleksey Shakhmatov ${ }^{3}$ and Robert Badrak ${ }^{4}$ \\ ${ }^{1 .}$ St. Petersburg State Polytechnical University/Metallurgical Technologies Department, St. Petersburg, \\ Russian Federation \\ 2. NPO TSNIITMASH, Moscow, Russian Federation \\ 3. Weatherford, St. Petersburg, Russian Federation \\ 4. Weatherford, Houston, Texas, USA
}

High nitrogen steels belong to special class of iron-based multicomponent alloys. Nitrogen is a strong austenite stabilizer and improves the mechanical and corrosion resistance properties. Nitrogen is also a relatively low cost alloying element for producing stainless steel.

The final stainless steel properties depend on the microstructure, the formation of which takes place at all stages of production, from the melting process, casting and solidification to plastic deformation and heat treatment. With that, the microstructure of stainless steel depends on chemical composition and evolves at each subsequent technological stage based on metallurgical heritage of structure formed during the solidification and it is known that austenitic steels could solidify by different modes and phase formation, including ferrite $[1,2]$.

The purpose of this work is observation and characterization of the as-cast structure of high nitrogen steels using optical metallography and thermodynamic simulation.

Specimens of high nitrogen steels were obtained by the method of fractional casting [3] related to making of small ingots with different chemical compositions (table 1). As-cast samples were studied in after annealing at $1060^{\circ} \mathrm{C}$ for $1 \mathrm{~h}$. Microstructures were revealed by tint etching using reagent with the following composition: $10 \mathrm{ml} \mathrm{HCl}+90 \mathrm{ml} \mathrm{H}_{2} \mathrm{O}+1 \mathrm{~g} \mathrm{Na}_{2} \mathrm{~S}_{2} \mathrm{O}_{5}$. Tint etching was performed using polarized light and filters that allows emphasizing structural and isolated fragments with dendritic arms which have the similar crystallographic orientation. Microstructural analysis was carried out with using a motorized light microscope Zeiss Axiovert 200 MAT, powered by image analyzer Thixomet. Software package FactSage with SGTE databases were used for thermodynamic simulation.

Fig. 1a shows an austenitic structure of specimen № 1 stainless steel, where all the austenite grains are formed by clear primary and secondary dendritic arms with the common crystallographic orientation. In figure 1b, the austenite structure of specimen № 2 does not contain ferrite, where austenite grains formed by primary and secondary dendritic arms, but some of them have a "blurring effect" where some grains have a blurred dendritic pattern. Figure 1c shows a duplex structure of specimen №3 with about $5 \%-10 \%$ ferrite phase and in this case dendritic arms in austenite grains are not observed.

According to the results of simulation (fig.2) specimen № 1 solidifies with only primary austenite phase and ferrite phase did not form in the region of annealing temperature $1060^{\circ} \mathrm{C}$. Specimen № 2 shows no ferrite at the annealing temperature, and the solidification process was accompanied by the formation of primary austenite with formation of ferrite phase. Specimen № 3 was solidified with primary ferrite and secondary austenite. Also, ferrite phase does exist (about 5\%) at the annealing temperature. 
By summarizing we can conclude that the ferrite formed during the solidification process, and further it's polymorphic transformation leads to a concentration gradient due to the different solubility of alloying and impurity elements in the ferrite and austenite. Because of this gradient, the elements diffuse to the depletion region of austenite that was formed from ferrite. The solidification with austenite only fixes chemical inhomogeneity, whereas solidification with ferrite, and the further transformation of ferrite to austenite leads to a "blurring" of a dendritic structure and homogenizing of chemical composition while cooling the solidified steel and in the process of heat treatment.

\section{References:}

[1] K. Rajasekhar et al, Materials Characterization, 38 (1997), Issue 2, p. 53

[2] M. A. Martorano et al, ISIJ International, 52 (2012), №. 6, p. 1054

[3] A.V. Shahmatov et al, Tyazheloe Mashinostroenie Journal, №. 2-3 (2014), p.3

\begin{tabular}{|c|c|c|c|c|c|c|c|}
\hline Specimen & $\mathrm{C}$ & $\mathrm{Si}$ & $\mathrm{Cr}$ & $\mathrm{Mn}$ & $\mathrm{Ni}$ & $\mathrm{Mo}$ & $\mathrm{N}$ \\
\hline 1 & 0.06 & 0.48 & 20.56 & 24.32 & 1.50 & 1.23 & 1.20 \\
\hline 2 & 0.06 & 0.44 & 20.59 & 23.94 & 2.19 & 1.11 & 1.09 \\
\hline 3 & 0.06 & 0.36 & 22.78 & 25.98 & 2.65 & 0.35 & 0.99 \\
\hline
\end{tabular}

Table 1. Chemical compositions of investigated specimens, \%
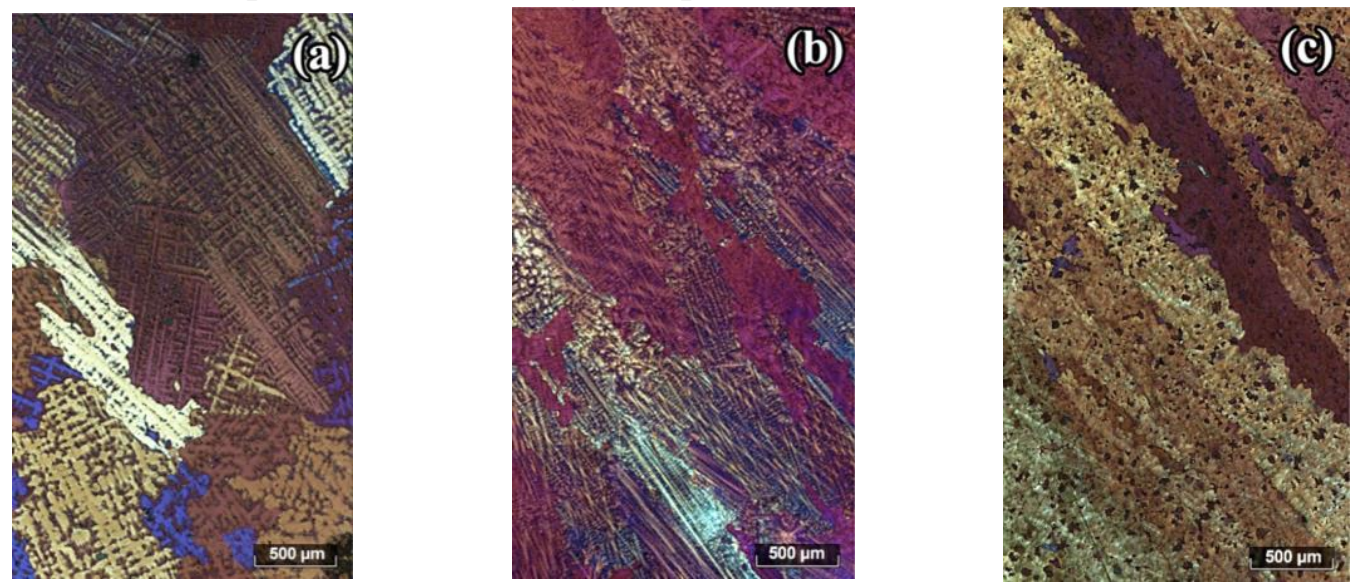

Figure 1. As-cast structure of steel specimens: a, b, c - specimen № 1, № 2, № 3, tint etched, polarized light, sensitive tint, $\times 50$

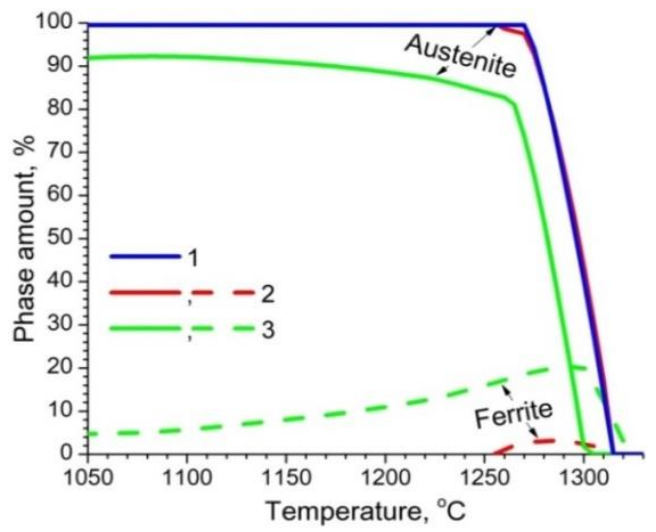

Figure 2. Sequence of phase formation in investigated specimens: №1(1), №2(2), №3(3) 